\title{
Distribution of binary mergers around galaxies
}

\author{
Tomasz Bulik and Krzysztof Belczyński
}

\author{
Nicolaus Copernicus Astronomical Center, Bartycka 18, 00-716 Warszawa,Poland
}

\begin{abstract}
We use a stellar binary population synthesis code to find the lifetimes and velocities of several types of possible GRB progenitors: double neutron stars, black hole neutron stars, black hole white dwarfs, helium star mergers. Assuming that they are born in different types of galaxies we compute their spatial distribution and compare it with the observed locations of GRB afterglows within their hosts. We discuss constraints on the compact object merger model of GRBs imposed by this comparison and find that the observations of afterglows and their host galaxies appear inconsistent with the GRB compact object merger model.
\end{abstract}

\section{INTRODUCTION}

In the last few years the astronomical community has moved much closer to unveiling the nature of GRBs. The discovery of afterglows and identification of host galaxies for several bursts clearly links GRBs to some type of stellar events. Yet the nature of these events is unknown, and we still do not know what the GRB central engines are. Observations of GRB host galaxies and precise locations of GRBs within hosts provide a tool to test some of the possible central engine models. In this paper we discuss the consistency between the current observations and the results of binary population synthesis.

\section{THE MODEL}

The population synthesis code used here is described in [3]. One of the most important parameter deterimining the properties of the populations of binaries is the kick velocity a newly formed compact object receives at birth. However, several studies $[4,7]$ indicate that the distribution of kick velocities consists of two components: a low velocity with the width of approximately $200 \mathrm{kms}^{-1}$, and a high velocity with the characteristic velocity around $800 \mathrm{kms}^{-1}$. About $80 \%$ of the kicks are drawn from the first component. It is also known that the production rate of compact object binaries falls off exponentially with increasing kick velocity, see Fig. 1 in [3]. Thus the population of compact object binaries will be dominated 
by the objects formed in the systems that received the kicks drawn from the low velocity component of the distribution. In the following we will consider the properties of the compact object binaries for the case when the kick velocity is drawn from a Gaussian distribution with the width $200 \mathrm{kms}^{-1}$.

Little is known a priori about the masses and gravtational potentials of host galaxies where GRB progenitrs reside. Therefore to find the expected distribtion of merger sites around galaxies we consider two extreme cases: propogation in a potential of a large galaxy like the Milky Way and propagation in the empty space [2].

\section{RESULTS}

In Figure 1 we present the distribution of center of mass velocities gained by systems in the supernova explosions in the galactic potantial and binary lifetimes (the time binary takes to evolve from ZAMS to final merger of two components) for four types of compact object binaries.

For the case of propagation in a potential of a massive galaxy only a fraction of NS-NS binaries will be able to escape from their host galaxies. The BH-NS binaries tend to stay in the galaxy. Here, we have assumed that the kick velocity does not depend on the nature of the compact object formed in the supernova. However, it has been argued that the kicks back holes receive should be smaller than those of the neutron stars. This is discussed in more detail in [1]. The helium star mergers stay in the host galaxies, while some of white dwarf black hole mergers have a chance of taking place outside the host, provided that the escape velocity from a given galaxy is not too large.

In the case of propagation in empty space quite a large number of binaries of any type will be able to escpae from their birthplace.

In Figure 2 we present the cumulative distributions of the distances (projected on the sky) between mergers sites and the host galaxies. In the case of propagation in empty space (left panel of Figure 2) most of the mergers take place far outside the host. In the case of propagation in the potential of a massive galaxy (right panel of Figure 2) black hole neutron star mergers and helium star mergers take place inside the host, and only a small, but not negligible fraction of double neutron stars and black hole white dwarf mergers happen outside the host.

\section{DISCUSSION}

We have learned at this conference [6] that the afterglow locations coincide with galaxies, and that typically there are intense star forming processes in these galaxies.

In the compact object merger model of GRBs one has to take into account the fact there are significant delays between the stellar formation and the time of merger. This delays consists of the stellar evolutionary time leading to supernavae 

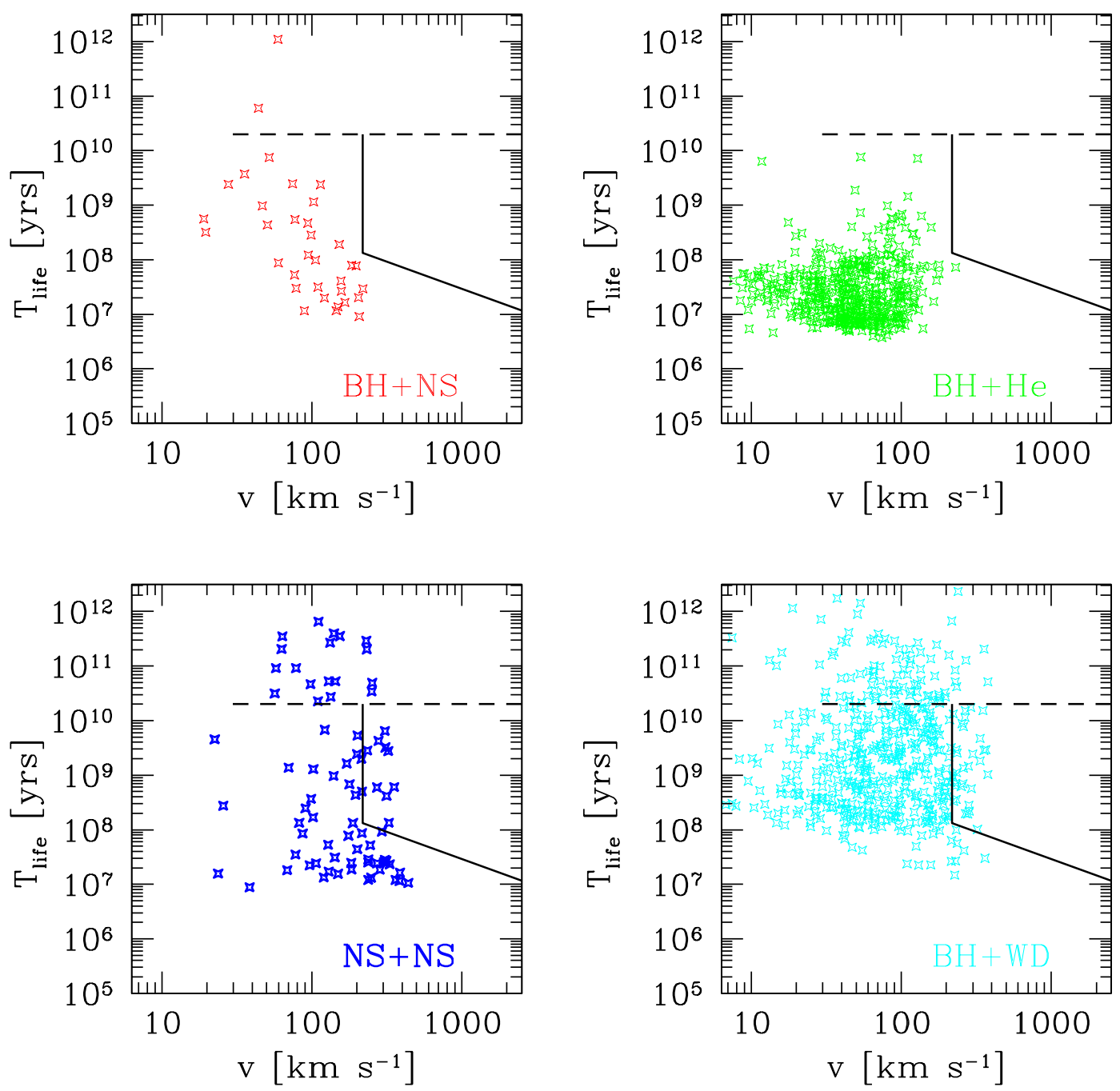

FIGURE 1. The distribution of different type of compact object binary mergers in the space spanned by the binary center of mass velocity and the binary lifetime (from ZAMS to final merger). The horizontal dashed line corresponds to the Hubble time (15Myrs). In the region for $t_{\text {merge }}<15$ Myrs we present two solid lines: the vertical corresponding to $v=200 \mathrm{~km} \mathrm{~s}^{-1}$ approximately the escape velocity from a galaxy, and the line corresponding to a constant value of $v \times t_{\text {merge }}=30 \mathrm{kpc}$. Together these lines define the region in the parameter space with systems that can escape from the host large galaxy.

explosions and formation of the compact onbject binary and the evolution of the compact object binary due to gravitational wave energy loss. The distribution of the delay times is rather wide and varies for different types of binaries [3], see Figure 1. In the case of helium star mergers these delays can be as short as a few 

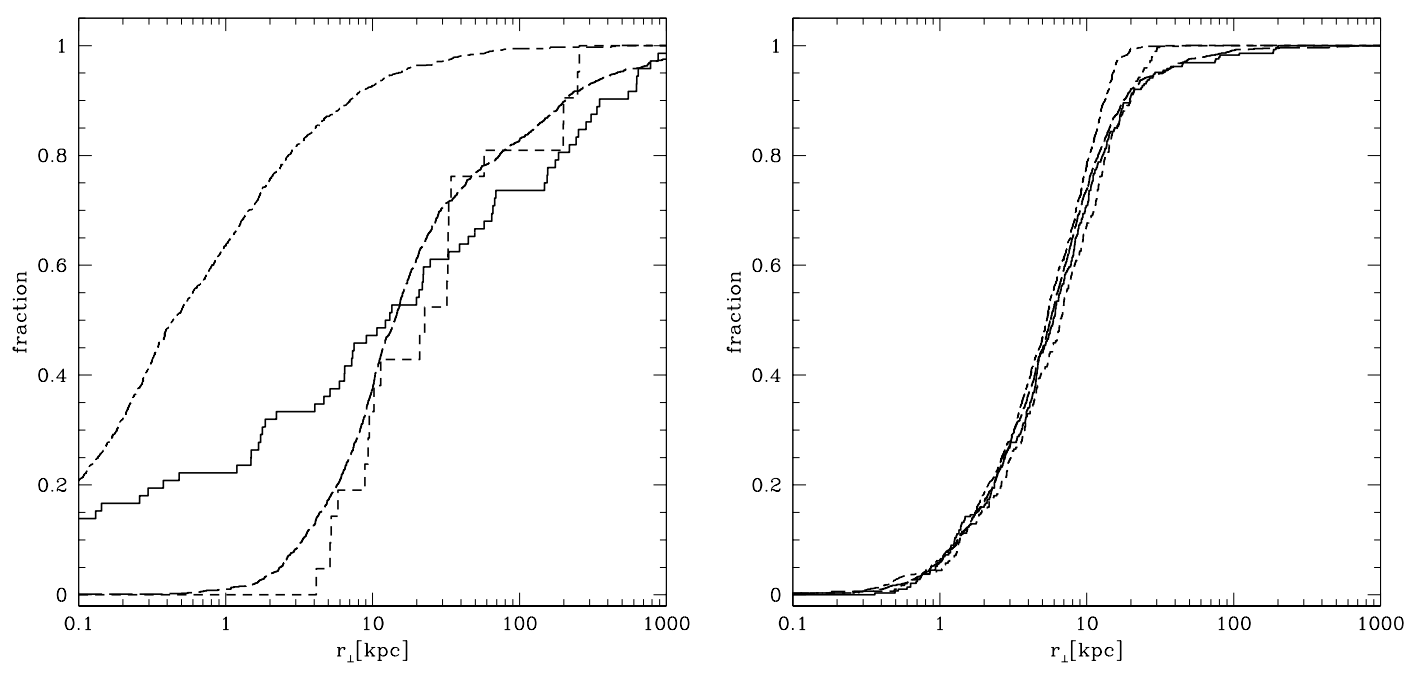

FIGURE 2. Distributions of different types of compact object binaries around their hosts. Left panel shows the case of prpagation in empty space and the right panel shows the case of propagation in a potential of a massive galaxy. The solid, short-dashed, long-dashed and dashed-dotted lines correspond to NS-NS, BH-NS, WD-BH, and He-BH mergers respectively.

million years.

Assuming that GRBs are related with NS-NS, BH-NS, or BH-WD mergers we do not expect any correlation between the GRB sites and star formation because the star formation processes could have ceased by the time the merger happens. Thus, within this model GRB rate should be proportional to the luminous mass in the Universe. As most of the luminous mass is concentrated in massive galaxies we expect to find GRBs within such galaxies. However, this should be typical galaxies and we tend to find GRB hosts in small, star forming ones [6].

Let us now consider the case when the delays between the stellar formation and the merger events are shorter than the star forming episode itself. Naturally, a correlation between the GRB sites and star forming galaxies exists. However, the observed host galaxies in this case are typically small. Thus, as shown above, a significant fraction of the mergers should take place outside the host galaxies and we should be finding GRBs with no underlying host galaxies.

One can also argue that the GRBs that take place outside the host galaxies do not produce significant afterglows, because of low density of the ambient medium and therefore are not followed by afterglows. This would make a strong selection effect against detecting GRBs happening outside of the hosts. At this conference we have heard however, that the Beppo SAX data are consistent with all GRBs having X-ray afterglows [5]. This means that all GRBs have afterglows and that GRBs with no afterglows do not exist, at least within the sample observable by Beppo SAX.

The reasoning presented above strongly argues against the compact object merger 
model of GRBs. However, we must remember that afterglows have been detected only from the long bursts, and we do not know if short bursts also produce afterglows and what are the locations in relation to host galaxies of short bursts. Moreover, numerical models of compact object coalescences $[8,9]$ agree with analytical estimates and show that the timescales of these events can not be stretched

beyond a fraction of a second. Yet the long bursts have a median duration of approximately $\approx 20-30 \mathrm{~s}$.

Thus we conclude that the compact object merger model appears to be inconsistent with the observations of afterglows, and their locations within the host galaxies. Long bursts are therefore most probably not connected with compact object mergers. However, it is quite likely that we will find that the short bursts are connected with mergers of compact objects.

Acknowledgments. We acknowledge the support of the following grants: KBN2P03D01616, KBN-2P03D00415.

\section{REFERENCES}

1. Belczynski K., Bulik T. and Zbijewski W., A\&A accepted, astro-ph/9911435 (1999).

2. Belczynski K., Bulik T. and Zbijewski W., MNRAS 309, 629 (1999).

3. Belczynski K., Bulik T. and Rudak B., 2000, this volume.

4. Dewey R.J., Cordes J.M., ApJ 321, 780 (1987).

5. Costa E., 2000, this volume.

6. Fruchter A., 2000, this volume.

7. Fryer C.L., Burrows A., Benz W., ApJ 496, 333 (1998).

8. Lee W.H. and Kluźniak W., Acta Astronomica 45, 705 (1995).

9. Ruffert M., Janka H.T., Takahashi K. and Schaefer G., A $\& A$ 319, 122 (1997). 